\title{
Application of Finite Element Analysis in Biomechanical Research of Degenerative Diseases of Lumbar Spine
}

\author{
Shuyi Zhang1, Tianyi Bai1, Xingxu Zhang2, Chao Feng³ ${ }^{3}$ Zhengpeng Liu1, Yilong Zhang1* \\ ${ }^{1}$ Department of Spine Surgery, The Affiliated Hospital of Chengde Medical College, Chengde, China \\ ${ }^{2}$ Beijing Pinggu Hospital, Beijing, China \\ ${ }^{3}$ Chengde Central Hospital, Chengde, China \\ Email: ${ }^{\star 952714988 @ q q . c o m ~}$
}

How to cite this paper: Zhang, S.Y., Bai, T.Y., Zhang, X.X., Feng, C., Liu, Z.P. and Zhang, Y.L. (2022) Application of Finite Element Analysis in Biomechanical Research of Degenerative Diseases of Lumbar Spine. Journal of Biosciences and Medicines, 10, 21-33.

https://doi.org/10.4236/jbm.2022.103004

Received: January 20, 2022

Accepted: March 1, 2022

Published: March 4, 2022

Copyright $\odot 2022$ by author(s) and Scientific Research Publishing Inc. This work is licensed under the Creative Commons Attribution International License (CC BY 4.0).

http://creativecommons.org/licenses/by/4.0/

\begin{abstract}
As the elderly population continues to grow, the number of patients with low back pain is gradually increasing. Among them, Lumbar Degenerative Diseases (LDD) is one of the major contributors to low back pain. Biomechanical in vivo studies of the lumbar spine are mainly performed by implants or imaging data to record the real-time changes of form and stress on the intervertebral disc during motion. However, the current developments are slow due to the technological and ethical limitations. In vitro experiments include animal experiments and cadaver experiments, which are difficult to operate or differ greatly from normal human structures, and the results still need to be verified repeatedly to test their accuracy. As for finite element method, it is relatively low cost and can repeat the experimental results. Therefore, we believe that finite element analysis plays an extremely important role in biomechanical research, especially in analyzing the relationship between different surgical models and the degeneration caused by different mechanics.
\end{abstract}

\section{Keywords}

Biomechanics, Degenerative Diseases of the Lumbar Spine, Animal Specimens, Human Cadaver Models, Finite Element Analysis, Statics Analysis, Stress, Range of Motion (RoM)

\section{Introduction}

At present, the aging of the whole society is gradually intensified, and the number of lumbar diseases is gradually increasing. Lumbar Degenerative Diseases (LDD) is one of the main causes of Lumbar and leg pain, and the treatment cost 
for Lumbar Degenerative Diseases is increasing year by year around the world, and the cost is huge [1] [2] [3]. In the process of aging and degeneration, the degeneration of lumbar spine and its corresponding muscle ligament structure is a normal physiological process [4]. In daily life, there are many reasons for lumbar degenerative diseases, such as sitting, standing and driving for a long time, etc. At present, the publicity for prevention and treatment of lumbar degenerative diseases is not enough, and the public lacks the awareness of protection, leading to lumbar degenerative diseases becoming more and more common [5]. Epidemiological studies show that more than half of people have experienced symptoms of low back discomfort in their lifetime, and in many studies, it even reached $84 \%$ [6]. In the face of complicated lumbar diseases that need to be solved urgently, the spine surgery department conducts in-depth studies on lumbar surgery, lumbar anatomy, lumbar injury mechanism and other aspects by using lumbar biomechanics [7]. Because in vivo experiments are limited by ethics and current technology, human cadaver model experiments and animal studies are costly and have a low repeatability rate, so they are less accepted by clinicians. Finite Element Method (FEM) is also known as Finite Element Analysis (FEA), which simulates the real physical system (geometry and load conditions) by using mathematical approximation. By using simple and interacting elements (i.e. units), a finite number of unknowns can be used to approximate a real system with infinite unknowns [8]. At present, it is widely used in clinical research, mainly because of its reproducible experimental results and low experimental cost, and it is widely used in the field of orthopedics and stomatology. Finite element analysis of spine mechanics has great advantages. It can not only study the mechanical etiology of degenerative lumbar degenerative disease, but also analyze the impact of different diagnoses and treatment methods on lumbar mechanics, or explore the mechanical principle of degenerative changes of adjacent segments caused by different diagnoses and treatment methods [9]. Therefore, finite element analysis can provide targeted preventive advice for healthy people or individuals with potential disease, especially for high-risk occupations causing lumbar disease. The research population can improve the internal fixation better. Each patient is provided with a personalized diagnosis and treatment plan [10]. This article mainly reviews the progress of the biomechanics of lumbar degenerative diseases and related fusion [1] [2]

\section{Biomechanical Study of Spine}

Biomechanical research can be roughly divided into two categories: outside the body and inside the body. In vitro studies can be divided into animal or cadaver studies and finite element analysis, which has been widely used in recent years. WILKE et al. [11] found in the study that the cervical spine of sheep is quite different from human, but the thoracic and lumbar vertebrae are similar to human, which can be used as the model of thoracic and lumbar vertebrae to replace human thoracic and lumbar vertebrae. KUMAR et al. [12] found that the thoracolumbar and lumbar spine of deer are very similar to human body, and can be 
used for mechanical experiments as long as the thoracolumbar spine of deer passes practical verification. However, due to the high price of animal experiments, the high diversity of some individuals, the failure to replicate the results, animal ethics, and the applicability of the conclusions, the conclusions need to be further proved. As for human cadaver experiments, the partial results and conclusions measured in these studies are widely regarded as the gold standard for other spinal biomechanical experiments [13]. Biomechanical analysis can analyze the impact of work and lifestyle on spinal degeneration [14] [15]. The current biomechanical disadvantage is that it cannot simulate the effect of human muscles on spinal movement in a realistic way [16]. In vivo studies were performed using a variety of imaging and mechanical monitoring devices to obtain a holistic and segmental spinal motion analysis [16] [17] [18]. However, each different research method has its own disadvantages. With the appearance of finite element analysis, the shortcomings of these studies are gradually made up in many aspects.

\section{Development of Finite Element Analysis in the Field of Spine Surgery}

In 1972, Belytschko et al. [19] used finite element analysis to analyze spine mechanics for the first time and constructed two-dimensional intervertebral disc structure, symbolizing the beginning of finite element method in spine biomechanical analysis. In 1975, Liu et al. [20] constructed a THREE-DIMENSIONAL lumbar spine model for the first time by using finite element method. Hkami and King [21] established the posterior structure and added it to the single-segment lumbar model, and analyzed the lumbar biomechanics related to dynamics and statics. The structural shape, material properties and load boundary conditions of the spine can all be simulated by using mathematical models in finite element analysis to measure the mechanical impact of changing relevant parameters on the overall structure [22]. At present, finite element method can be used in many fields of human body research, many of which are researches on biomechanical effects of implants on human body [23] [24]. A large number of scholars have used finite element method to set different loads to analyze the mechanics of spine physiology or pathology, as well as the biomechanical effects of different implants on spine. These data can enable researchers to improve implants and clinicians to make more reasonable spinal surgery plans for patients [25]. Compared with other biomechanical experiments, finite element analysis has the characteristics of low cost, repeatable conclusion and more efficiency, and has greater advantages over other biomechanical research methods. Finite element analysis can analyze the internal mechanics of vertebral body, disc and other tissues, which cannot be measured by other experimental methods [9].

\section{Principles of Finite Element Analysis for Degenerative Diseases of the Lumbar Spine}

The characteristics of the lumbar spine are irregular shape, heterogeneous 
structure, and the use of traditional biomechanical experiments to solve some of its biomechanical characteristics of the relatively difficult, finite element analysis can be very good traditional experiments cannot solve mechanics calculation, such as internal pressure of intervertebral disc, at the same time, can give different organizations give different material properties. An object can be decomposed into a large number of particles. The total number of these particles approaches to infinity, which gives them infinite degrees of freedom. Therefore, mechanical analysis cannot be carried out for them [26]. The invention of the finite element analysis, we can on a computer modeling of arbitrary objects, set to become a finite number of units of the model, gives objects related material parameters, the model only through each node transition, because nodes are relatively limited, means that the degrees of freedom are relatively limited, which makes the model can be obtained relatively. It can be transformed into a linear algebraic continuous structure, and the mechanical results can be solved through relevant formulas or software operations, which is called finite element method [27].

\section{Method of Establishing Lumbar Spine Model with Finite Element Technique}

At present, there are many methods to obtain experimental data by finite element analysis, such as: 1) 3D coordinate instrument modeling method: obtain the coordinates of objects in $3 \mathrm{~d}$ space and solve them node by node. The solving process is tedious, but the data is accurate [28]. 2) Geometric modeling: Proportional modeling of object shape is adopted, which is mostly used for objects with regular shape [29]. 3) Image modeling: Imaging data of lumbar spine were obtained through CT and MRI, and finite element model was constructed through 3D modeling software [28]. Compared with other models, the model constructed by this method is more simulation. At present, with the continuous improvement of the accuracy of thin-layer CT and MRI, the finite element model created can better reflect the differences of everyone's lumbar spine, and the results calculated by software can better reflect the different situations between individuals, which also makes image modeling become the mainstream finite element modeling method [30].

\section{Advantages and Limitations of Different Biomechanical Methods of Lumbar Spine Research}

In vitro biomechanical research methods can be classified into two types, one is through cadaver or animal experiments, the other is to create a 3D model. Cadaver experiments and animal experiments have their own limitations: cadaver specimens are relatively difficult to obtain, and most cadaver bones are lost with bone loss, which leads to many cadaver bone models that cannot represent the bones of normal adults, and cadaver experiments have problems with muscle and intervertebral disc control [31]. At present, there are relatively few studies on spinal mobility through cadaver experimental technology, mainly because it 
is relatively difficult to simulate the degree of degeneration and the technology is complicated [26] [31]. However, the intervertebral disc in animal models is different from the human body and can provide limited information on the pathophysiology of human diseases under different loads. In vivo experiments can obtain the overall situation of the human body under various load changes, the mechanical changes of the disc are relatively complete, but the compression and nutritional supply mechanism is damaged, and plays a key role in the pathogenesis of the disc. However, studying the relationship between nutrient loss in intervertebral discs and compressed intervertebral discs has been difficult in vivo, especially in human intervertebral discs [32]. Therefore, finite element analysis can serve as an important supplement to help us fully understand the dynamics of the internal motion of the disc. Through finite element analysis, some researchers have found that compression affects nutrient distribution. Specifically, dynamic compression increases oxygen concentration and reduces lactic acid accumulation. On the contrary, static compression has opposite effects on oxygen and lactic acid transport and metabolism [33]. But evidence from human trials is lacking.

The common limitation of any in vitro experiment is the variability of the material properties associated with the spine. For example, the water content of the disc changes with age and the material properties vary. Iatridis et al. [34] studied the elasticity of nucleus pulposus and annulus fibrosus at different ages, and intervertebral disc elasticity would deteriorate with age. In vitro experiments generally remove muscle tissue, which sacrifices its role in maintaining global stability of the spine as a whole, and it is impossible to alter the movement of the spine through loading. The intervertebral disc acts as a cushion against statics, shock, and cyclic vibration, and the moisture inside the disc plays a key role. In vitro experiments, when he stopped counting the moisture in the disc from loading to loading, the results were different, leading to changes in the recovery time of the spine after movement, resulting in different mechanical results from the ideal state. These will lead to differences in the calculation results of different experiments [26].

\section{Characteristics of Lumbar Biomechanics through Finite Element Analysis}

At present, many scientists verify the validity of the experimental results of finite element analysis through finite element analysis and experimental comparative study, proving that the established model is a usable model [35]. Kasra et al. [36] conducted finite element analysis and experiment on lumbar spine at the same time, and no significant difference was found in the results obtained. Compared with other biomechanical experiments, finite element analysis can solve the stress or contact friction of the contact surface of different objects by setting the actual material parameters and adding the corresponding control load through computer. These results are difficult to be calculated through experiments. The 
finite element analysis software splits the lumbar spine model into a limited number of nodes. Through the mechanical operation analysis of these nodes, the biomechanical results of the lumbar spine can be solved, and a large number of results that cannot be solved by experiments can be provided, such as the internal mechanical situation of the object, such as the stress and strain of the intervertebral disc. In the direction of spine research, the results of finite element analysis can be repeatable. Different parameters can be set to obtain the mechanical condition of the desired parts, and even mechanical analysis can be carried out on a certain surface and point. Finite element analysis can analyze the mechanical changes of lumbar degeneration and postoperative mechanical changes, so as to provide suggestions for clinical surgical design and internal fixation device optimization. Although most finite element models are constructed by relatively objective imaging data such as INDIVIDUAL lumbar CT or nuclear magnetic resonance, they still need to complement traditional experiments. By the limits of science and technology, is still a big rise space finite element analysis, the structure of the spine is complicated, and our model assumes that or is a numerical model is simplified [37], it can only represent a small part of the spine, and not for each different material parameters in the model, instead of only close to the real situation, Therefore, the results and conclusions need to be supplemented with clinical practice.

For promotion and further lumbar spinal finite element analysis, finite element analysis for lumbar spine surgeon and researcher at the research of the main study of the characteristics of the static mechanics, there is also a part of the dynamic mechanical properties, instrument implant, the vibration damage, tissue damage, the relationship between the related muscles and degeneration problem, can for different people, professional set suitable operation method. There have also been several studies on the degradation and injury of the lumbar spine associated with vibration environment from an ergonomic perspective. Liu Jie [38] through the finite element analysis, such as people in the low frequency vibration of vehicle, different length for various degenerative degree of the influence of human lumbar spine biomechanics, the low frequency vibration, short time and with various degrees of degeneration of the lumbar spine damping vibration reduction effect is good, when lumbar by vibration for a long time, moderately severe degeneration of the lumbar spine mechanics performance obvious variation, Patients with severe degeneration are advised to reduce driving time. Shu Xiantao and Li Xiaolin [39] analyzed the dynamic mechanics of the anterior longitudinal ligament during the hyperextension reduction of patients with thoracolumbar compression fractures by using the finite element method, and described that when the hyperextension fulcrum is at the apex of the spinous process of the injured vertebra, the reduction is the most complete and the mechanical properties of the anterior longitudinal ligament are the most stable. In the process of simulated reduction, the anterior longitudinal ligament is nonlinear. Huang Ping et al. [40] derived the process of lumbar degenerative scoliosis through finite element method, as well as biomechanical changes and dege- 
nerative lumbar intervertebral disc, which would lead to the reduction of the height of the nucleus pulposus and the increase of the load on the annulus fibrosus, resulting in the deformation of the intervertebral disc, indirectly leading to the increase of the stress of vertebral appendages and the reduction of the stress of the nucleus pulposus [40]. Arnoux P J, et al. [41] found that the impact of nucleus pulposus degeneration on lumbar mechanics was significantly greater than that of annulus fibrosus degeneration. After nucleus pulposus degeneration, the intervertebral disc hardened, material parameters changed significantly, and lumbar biomechanics changed. Ding Jinyong et al. [42] using finite element method (fem) of lumbar sacral 5-1 segment joints through the research of structural abnormalities in flexion and rotation under load, joints asymmetry can lead to stress of the vertebral body was unreasonable situation, under the former flexion and rotation motion, different Angle of the joints, will have different dish of internal stress, at the same time, the contact force of joints, joint will be different. Tang Liang et al. [43] determined the related factors of lumbar injury from the perspective of biomechanics by applying response load and impact load to the lumbar spine. When the impact load was applied, the bent lumbar spine had less risk of injury compared with the independent lumbar spine, while when the response load was applied, the ligament relaxation area and response area had greater risk of injury.

\section{The Role of Finite Element Analysis in the Treatment of Lumbar Degenerative Diseases}

At present a lot of different internal fixation devices and the emergence of a new mode of operation to need a lot of retrospective study or related to validate its biomechanical experiments, such as Fan and Guo [44], through the anterior, posterior and the intervertebral foramen lumbar fusion between biomechanical comparison of the impact of human lumbar vibration characteristic, found in dynamic response, The endplate stress of fusion segments with transforaminal approach was lower than that with anterior and posterior approaches, but the pedicle screw stress was higher in the transforaminal model than in the anterior and posterior models. Fan and Guo et al. [45] studied the role of posterior screw fixation in single-segment transforaminal lumbar interbody fusion during whole body vibration through finite element analysis, and found that bilateral pedicle screw fixation could well avoid spinal injury caused by vibration. Jia et al. [46] conducted harmonic response of simulated vehicle vibration frequency in patients with scoliosis through wired meta-analysis, and found that scoliosis was more sensitive to vibration forms. When the frequency of lumbar axial cyclic vibration load is close to its resonance frequency, the vibration amplitude is large. These results suggest that vibration exacerbates the severity of scoliosis and should therefore be reduced in such patients. Clinical treatment should pay attention to scoliosis and reduce its vibration. These findings may contribute to the clinical prevention and treatment of scoliosis. These vibration models can be helpful in the treatment of patients in professions such as driving vehicles. 
For many new surgery and internal fixation can also through the finite element analysis, clear its stability, such as Chen et al. [47] by using the finite element analysis to improve lumbar vertebral fusion between vertebral lateral fusion (CLIF) biomechanical research, analysis of this new fusion should be put on the epiphyseal ring, in order to obtain suitable biomechanical stability, Bilateral pedicle screws should also be used to reduce sink of the fusion apparatus. Jiang et al. [48] compared the new lumbar posterior dynamic stabilization system with the traditional internal fixation system through finite element analysis, and found that the new posterior dynamic stabilization system could better make the activity of the lesion segment close to normal, and reduce the increased activity of the adjacent segment and the stress of the intervertebral disc and facet facet of the adjacent lumbar spine. These conclusions show that the new device can meet the design requirements. Guo et al. [49] analyzed the optimized CoFlex using finite element analysis method. Compared with the traditional CoFlex-F, the new interspinoid spinoid spitter can achieve better stability and reduce the stress of the implanted device. Jiang [50], such as research into the way of intervertebral fusion after impact on the degeneration of adjacent segments, relative to the normal model, the adjacent vertebral degeneration results in the decrease of relative motion of adjacent segments, intervertebral disc and annulus pressure to increase, so the adjacent segment degeneration is lumbar posterior arthrodesis postoperative vertebral disease risk factors. Zhang et al. [51] used finite element analysis to compare bilateral pedicle screws with cortical screws and found that, in the fixed segment, cortical bone path east may provide better stability, endplate tension and facet joint stress than bilateral pedicle screws. However, cortical bone screws have relatively large range of motion, endplate stress and facet stress in adjacent segments, which should be considered comprehensively in the selection of future treatment methods.

For many patients with scoliosis. Guan et al. [52] studied the finite element analysis of three-dimensional correction branch of adolescent idiopathic scoliosis, and designed and optimized support frames through finite element analysis to achieve better correction goals. This method can effectively improve the efficiency and treatment effect of correction design and optimization, and this external brace can avoid surgical treatment for some patients and reduce the patient burden. Kumaran et al. [53] analyzed the influence of open and minimally invasive surgery on muscle injury and degeneration of adjacent segments through finite element analysis. The reduction of the paraspinal muscle cross-sectional area of the spine can lead to aggravation of degeneration of adjacent segments, leading to postoperative continuous back pain, and minimally invasive surgery can reduce muscle loss [54]. Newcomb in combination with body experiment and finite element analysis techniques, they compared the different pedicle screws in the lumbar spine. Angle of nailing screw stress changes, the results show that the screw and sagittal axis Angle, the greater the screw, the smaller the stress calculated by large Angle pedicle screw implantation and can significantly reduce the risk of screw breakage and loosening of the conclusion. Chen ZHAO- 
hui's team [55] analyzed and compared the differences of stability, intervertebral disc and facet joint stress between the two models by establishing lumbar interspinous process distraction and pedicle screw fixation model. It was proved that the Coflex splitter could significantly reduce the intervertebral stiffness of the fixed segment, have little intervention on the range of motion of the responsible segment, and reduce the stress values of joints and discs. Except for the increase of the stress values of the facet joints of the upper and lower segments, the above-mentioned indexes of the adjacent segments had little change.

\section{Conclusion}

Finite element technique has the characteristics of convenience and high repeatability of experimental results. There is also reason to believe that in the future, every physician, especially spinal surgeons, will be able to master finite element methods. Through personalized modeling of different patients, we can make targeted treatment plans for each patient, make more reasonable optimization of various orthopedic internal fixation devices, improve long-term efficacy, reduce the probability of complications in late patients, and analyze and deduce the correlation between lumbar degeneration and lumbar mechanical changes. At present, the finite element technology still has a lot of room for improvement, which needs us to improve. The overall model made by the current software is only a THREE-DIMENSIONAL model, which cannot reflect many specific details of the corresponding individual. The relevant software still needs a large number of scientists to improve and optimize, so as to reduce the difficulty of operation and promote it better. At present, lumbar diseases still need to be further combined with relevant clinical, animal experiments and follow-up studies, which can provide help for the diagnosis and treatment of the disease and provide a more reasonable explanation for the etiology research.

\section{Conflicts of Interest}

The authors declare no conflicts of interest regarding the publication of this paper.

\section{References}

[1] Andersson, G.B. (1999) Epidemiological Features of Chronic Low-Back Pain. The Lancet, 354, 581-585. https://doi.org/10.1016/S0140-6736(99)01312-4

[2] Martin, B.I., Deyo, R.A., Mirza, S.K., et al. (2008) Expenditures and Health Status among Adults with Back and Neck Problems. JAMA, 299, 656-664. https://doi.org/10.1001/jama.299.6.656

[3] Zhang, F.S. (2018) A Short-Term Clinical Study of Intervertebral Plate Dynamic Stability System Intra Spine and Traditional Fusion Surgery for Treatment of Lumbar Degenerative Diseases. Henan University of Chinese Medicine, Zhengzhou.

[4] Guo, H.Z. (2019) The Efficacy, Complications and Finite Element Analysis of stability of Lateral Interbody Fusion in Lumbar Spine Diseases. Guangzhou University of Chinese Medicine, Guangzhou. 
[5] Hou, Z.Y., Zhang, H. and Luo, T.H. (2017) Treatment of Degenerative Lumbar Spinal Stenosis: A Review. World Update Medical Information Abstract, 17, 38-39.

[6] Ge, H., Zhang, P., Sun, W.D., et al. (2021) The Mechanism of Fascial Manipulation and Its Application in Rehabilitation of Nonspecific Low Back Pain. Chinese Journal of Rehabilitation Medicine, 36, 237-240.

[7] Xu, J.M. and Li, J. (2002) Analysis of Causes of Failure Syndrome of Lumbar Spine Surgery in 58 Cases. Chinese Journal of Orthopaedic Surgery, No. 11, 86-87.

[8] Fan, Z.R. (2019) Finite Element Analysis and Measurement of Internal Declination Angle and Plantar Angle of Screw Internal Fixation for Talus Neck Fracture Based on 3D Reconstruction. Tianjin Medical University, Tianjin.

[9] Dreischarf, M., Zander, T., Shirazi-Adl, A., et al. (2014) Comparison of Eight Published Static Finite Element Models of the Intact Lumbar Spine: Predictive Power of Models Improves When Combined Together. Journal of Biomechanics, 47, 1757-1766. https://doi.org/10.1016/j.jbiomech.2014.04.002

[10] Sun, K. and Yang, X.J. (2019) Application of Finite Element Technique in Lumbar Degenerative Disease. Chinese Journal of Tissue Engineering Research, 23, 2553-2559.

[11] Wilke, H.J., Kettler, A., Wenger, K.H., et al. (1997) Anatomy of the Sheep Spine and Its Comparison to the Human Spine. The Anatomical Record, 247, 542-555. https://doi.org/10.1002/(SICI)1097-0185(199704)247:4\%3C542::AID-AR13\%3E3.0. CO;2-P

[12] Kumar, N., Kukreti, S., Ishaque, M., et al. (2000) Anatomy of Deer Spine and Its Comparison to the Human Spine. The Anatomical Record, 260, 189-203. https://doi.org/10.1002/1097-0185(20001001)260:2\%3C189::AID-AR80\%3E3.0.CO; $\underline{2-\mathrm{N}}$

[13] Guo, H.Z., Tang, Y.C., Guo, D.Q., et al. (2020) Stability Evaluation of Oblique Lumbar Interbody Fusion Constructs with Various Fixation Options: A Finite Element Analysis Based on Three-Dimensional Scanning Models. World Neurosurgery, 138, e530-e538. https://doi.org/10.1016/j.wneu.2020.02.180

[14] Cook, D.J., Yeager, M.S. and Cheng, B.C. (2015) Range of Motion of the Intact Lumbar Segment: A Multivariate Study of 42 Lumbar Spines. International Journal of Spine Surgery, 9, 5. https://doi.org/10.14444/2005

[15] White, A.R. and Panjabi, M.M. (1978) The Basic Kinematics of the Human Spine. A Review of Past and Current Knowledge. Spine, 3, 2-20.

[16] Zhao, P. and Zhao, Y. (2020) Research Progress of Finite Element Analysis in the Treatment of Lumbar Spine Diseases. World Latest Medical Information Abstracts (Continuous Electronic Journal), 20, 168-169.

[17] Pearcy, M.J. and Tibrewal, S.B. (1984) Axial Rotation and Lateral Bending in the Normal Lumbar Spine Measured by Three-Dimensional Radiography. Spine, 9, 582-587.

[18] Zafereo, J., Wang-Price, S., Brown, J., et al. (2016) Reliability and Comparison of Spinal End-Range Motion Assessment Using a Skin-Surface Device in Participants With and Without Low Back Pain. Journal of Manipulative and Physiological Therapeutics, 39, 434-442. https://doi.org/10.1016/j.jmpt.2016.05.008

[19] Belytschko, T.B., Andriacchi, T.P., Schultz, A.B., et al. (1973) Analog Studies of Forces in the Human Spine: Computational Techniques. Journal of Biomechanics, 6, 361-371. https://doi.org/10.1016/0021-9290(73)90096-1

[20] Liu, Y.K., Ray, G. and Hirsch, C. (1975) The Resistance of the Lumbar Spine to Direct Shear. Orthopedic Clinics of North America, 6, 33-49. 
https://doi.org/10.1016/S0030-5898(20)31198-6

[21] Hakim, N.S. and King, A.I. (1979) A Three Dimensional Finite Element Dynamic Response Analysis of a Vertebra with Experimental Verification. Journal of Biomechanics, 12, 277-292. https://doi.org/10.1016/0021-9290(79)90070-8

[22] Xie, W.X. (2019) Analysis of Risk Factors for New Vertebral Fractures after Vertebral Augment and Finite Element Study of Its Influence on the Mechanics of Adjacent Segments. Guangzhou University of Chinese Medicine, Guangzhou.

[23] Tsuang, F.Y., Hsieh, Y.Y., Kuo, Y.J., et al. (2017) Assessment of the Suitability of Biodegradable Rods for Use in Posterior Lumbar Fusion: An In-Vitro Biomechanical Evaluation and Finite Element Analysis. PLoS ONE, 12, e188034. https://doi.org/10.1371/journal.pone.0188034

[24] Huang, Y.P., Du, C.F., Cheng, C.K., et al. (2016) Preserving Posterior Complex Can Prevent Adjacent Segment Disease Following Posterior Lumbar Interbody Fusion Surgeries: A Finite Element Analysis. PLoS ONE, 11, e166452.

https://doi.org/10.1371/journal.pone.0166452

[25] Schmidt, H., Heuer, F. and Wilke, H.J. (2009) Dependency of Disc Degeneration on Shear and Tensile Strains between Annular Fiber Layers for Complex Loads. Medical Engineering \& Physics, 31, 642-649.

https://doi.org/10.1016/j.medengphy.2008.12.004

[26] Han, S. (2016) Application of Finite Element Analysis in Biomechanics of Lumbar Intervertebral Disc. Hebei Medical University, Shijiazhuang.

[27] Zhu, L. and Huo, H.J. (2014) Application of Finite Element Analysis in Kyphotic Deformity of Ankylosing Spondylitis. Chinese Medical Journal, 11, 166-168.

[28] Sun, K. (2019) Analysis and Study of Biomechanics after OLIF Using Finite Element Technique. Inner Mongolia Medical University, Hohhot.

[29] Chen, B.H., Sun, J., Peng, J. and Natarajan, N. (2002) An. Establishment and Significance of Three-Dimensional Finite Element Model of Cervical Spine. Chinese Journal of Spinal Cord, No. 2, 25-28.

[30] Tao, Y., Wu, Y.L., Zong, S.H., et al. (2016) Biomechanical Characteristics of Lumbar Internal Fixation Based on Finite Element Analysis. Chinese Journal of Tissue Engineering Research, 20, 1932-1938.

[31] Sunni, N., Askin, G.N., Labrom, R.D., et al. (2014) The Effect of Repeated Loading and Freeze-Thaw Cycling on Immature Bovine Thoracic Motion Segment Stiffness. Proceedings of the Institution of Mechanical Engineers, Part H, 228, 1100-1107. https://doi.org/10.1177/0954411914556789

[32] Smith, L.J., Nerurkar, N.L., Choi, K.S., et al. (2011) Degeneration and Regeneration of the Intervertebral Disc: Lessons from Development. Disease Models \& Mechanisms, 4, 31-41. https://doi.org/10.1242/dmm.006403

[33] Hu, B.W., Lv, X., Chen, S.F., et al. (2019) Application of Finite Element Analysis for Investigation of Intervertebral Disc Degeneration: from Laboratory to Clinic. Current Medical Science, 39, 7-15. https://doi.org/10.1007/s11596-019-1993-7

[34] Iatridis, J.C., Setton, L.A., Weidenbaum, M., et al. (1997) Alterations in the Mechanical Behavior of the Human Lumbar Nucleus Pulposus with Degeneration and Aging. Journal of Orthopaedic Research, 15, 318-322. https://doi.org/10.1002/jor.1100150224

[35] Ling, Q.J., Lin, H., Xie, P., et al. (2018) Establishment and Validation of Nonlinear Finite Element Model of Whole Lumbar Spine. Chinese Journal of Clinical Anato$m y, 36,662-668$. 
[36] Kasra, M., Shirazi-Adl, A. and Drouin, G. (1992) Dynamics of Human Lumbar Intervertebral Joints. Experimental and Finite-Element Investigations. Spine, 17, 93-102.

[37] Martinez, J.B., Oloyede, V.O.A. and Broom, N.D. (1997) Biomechanics of Load-Bearing of the Intervertebral Disc: An Experimental and Finite Element Model. Medical Engineering \& Physics, 19, 145-156. https://doi.org/10.1016/S1350-4533(96)00056-2

[38] Liu, J., Fan, R.X., Gao, J.Z., et al. (2020) Finite Element Analysis of Mechanical Response of Human Lumbar Spine with Varying Degrees of Degeneration under Low Frequency Vehicle Vibration. Chinese Journal of Tissue Engineering Research, 24, 1371-1377.

[39] Shu, X.T. and Li, X.L. (2009) Finite Element Analysis of Dynamic Mechanics of Anterior Longitudinal Ligament during Hyperextension Reduction in Patients with Thoracolumbar Compression Fractures. Chinese Journal of Tissue Engineering Research and Clinical Rehabilitation, 13, 9567-9569.

[40] Huang, P. (2013) Finite Element Analysis of the Evolution and Biomechanical Mechanism of Degenerative Lumbar Scoliosis. Second Military Medical University, Shanghai.

[41] El-Rich, M., Arnoux, P.J., Wagnac, E., et al. (2009) Finite Element Investigation of the Loading Rate Effect on the Spinal Load-Sharing Changes under Impact Conditions. Journal of Biomechanics, 42, 1252-1262. https://doi.org/10.1016/j.jbiomech.2009.03.036

[42] Ding, J.Y., Xu, J.X., Tan, C.S., et al. (2019) Abnormal Facet Joint Structure of Lumbar 5-SACRAL 1 in China. Chinese Journal of Science Journal of Traditional Medical Traumatology \& Orthopedics, 28, 23-28.

[43] Tang, L., Zheng, J.J., Li, W.X., et al. (2020) Biomechanical Model of Human Lumbar Spine and Sensitivity Analysis of Lumbar Spine Injury Parameters. Journal of South China University of Technology (Natural Science Edition), 48, 94-106.

[44] Fan, W. and Guo, L.X. (2019) Biomechanical Comparison of the Effects of Anterior, Posterior and Transforaminal Lumbar Interbody Fusion on Vibration Characteristics of the Human Lumbar Spine. Computer Methods in Biomechanics and Biomedical Engineering, 22, 490-498. https://doi.org/10.1080/10255842.2019.1566816

[45] Fan, W. and Guo, L.X. (2018) The Role of Posterior Screw Fixation in Single-Level Transforaminal Lumbar Interbody Fusion During Whole Body Vibration: A Finite Element Study. World Neurosurgery, 114, e1086-e1093. https://doi.org/10.1016/j.wneu.2018.03.150

[46] Jia, S., Li, Y., Xie, J., et al. (2019) Differential Response to Vibration of Three Forms of Scoliosis during Axial Cyclic Loading: A Finite Element Study. BMC Musculoskeletal Disorders, 20, Article No. 370. https://doi.org/10.1186/s12891-019-2728-4

[47] Chen, Y.L., Lai, O.J., Wang, Y., et al. (2020) The Biomechanical Study of a Modified Lumbar Interbody Fusion-Crenel Lateral Interbody Fusion (CLIF): A Three-Dimensional Finite-Element Analysis. Computer Methods in Biomechanics and Biomedical Engineering, 23, 548-555.

[48] Jiang, S., Liu, Y., Chen, Y.Y., et al. (2021) The Three-Dimensional Finite Element Analysis of the Dynamic Stable System of the New Lumbar Spine. Beijing Biomedical Engineering, 40, 24-30.

[49] Guo, L.X. and Yin, J.Y. (2019) Finite Element Analysis and Design of an Interspinous Device Using Topology Optimization. Medical \& Biological Engineering \& Computing, 57, 89-98. https://doi.org/10.1007/s11517-018-1838-8

[50] Jiang, S. and Li, W. (2019) Biomechanical Study of Proximal Adjacent Segment Degeneration after Posterior Lumbar Interbody Fusion and Fixation: A Finite Element 
Analysis. Journal of Orthopaedic Surgery and Research, 14, Article No. 135. https://doi.org/10.1186/s13018-019-1150-9

[51] Zhang, L., Li, H.M., Zhang, R., et al. (2021) Biomechanical Changes of Adjacent and Fixed Segments Through Cortical Bone Trajectory Screw Fixation versus Traditional Trajectory Screw Fixation in the Lumbar Spine: A Finite Element Analysis. World Neurosurgery, 151, e447-e456. https://doi.org/10.1016/j.wneu.2021.04.061

[52] Guan, T., Zhang, Y., Anwar, A., et al. (2020) Determination of Three-Dimensional Corrective Force in Adolescent Idiopathic Scoliosis and Biomechanical Finite Element Analysis. Frontiers in Bioengineering and Biotechnology, 8, Article No. 963. https://doi.org/10.3389/fbioe.2020.00963

[53] Kumaran, Y., Shah, A., Katragadda, A., et al. (2021) Iatrogenic Muscle Damage in Transforaminal Lumbar Interbody Fusion and Adjacent Segment Degeneration: A Comparative Finite Element Analysis of Open and Minimally Invasive Surgeries. European Spine Journal, 30, 2622-2630.

[54] Newcomb, A.G., Baek, S., Kelly, B.P., et al. (2017) Effect of Screw Position on Load Transfer in Lumbar Pedicle Screws: A Non-Idealized Finite Element Analysis. Computer Methods in Biomechanics and Biomedical Engineering, 20, 182-192. https://doi.org/10.1080/10255842.2016.1209187

[55] Chen, Z.H. (2010) A Finite Element Analysis and Clinical Study on Lumbar Interspinous Process Distraction Device Surgery. Second Military Medical University, Shanghai. 